\title{
Diagnosis and Treatment Experience of 68 Cases on Pediatric Acute Intussusception
}

\author{
Yongxing Huang* \\ Yanling Health Center, Danyang, Jiangsu 212300, China
}

\begin{abstract}
Objective: To provide reference for the accurate clinical diagnosis and successful treatment by analyzing the clinical experience of pediatric acute intussusception. Methods: Retrospective analysis of 68 cases of pediatric acute intussusception in the hospital between January 2007 and December 2014. Results: For the 68 cases of pediatric acute intussusception, first diagnosis as acute intussusception in 49 cases (72.1\%), first diagnosis as enteritis or bacillary dysentery in 16 cases $(23.5 \%), 3$ cases of unknown diagnosis (4.4\%). All patients in 68 cases were treated for air enema reduction, of which 35 cases were successful (success rate is 51.5\%), 33 cases were successful in operative reduction (success rate is $48.5 \%$ ). Conclusion: Clinicians should improve the understanding of pediatric acute intussusception, diagnose as early as possible, take timely air enema or surgical reduction; the probability of success of air enema reduction and surgical reduction were both high; improve the life environment and hygiene situations, the enhancement of life quality to an extent can reduce the occurrence of pediatric acute intussusception.
\end{abstract}

\section{KEYWORDS}

Pediatric intussusception

Air enema

Operative reduction

Diagnosis experience

\section{Introduction}

Pediatric acute intussusception is a common pediatric acute abdominal disease, whose clinical manifestations are abdominal pain (paroxysmal crying), vomiting, currant jelly blood stool and abdomen mass, which can lead to intestinal necrosis, infectious shock even death in severe cases, and its etiology and pathogenesis is not yet fully understood [1]. The therapeutic methods include air enema reduction and surgical reduction with high success rates. This study retrospectively analyzed 68 cases of pediatric acute intussusception in the hospital between January 2007 and December 2014, and summarized the diagnosis experience as follows.

\section{Clinical data \\ 2.1. General data}

Copyright ( 2015 Yongxing Huang

doi: $10.18686 /$ jn.v4i2.3

Received: February 15, 2015; Accepted: April 21, 2015; Published online: June 27, 2015

This is an open-access article distributed under the terms of the Creative Commons Attribution Unported License (http://creativecommons.org/ licenses/by-nc/4.0/), which permits unrestricted use, distribution, and reproduction in any medium, provided the original work is properly cited.

${ }^{\star}$ Corresponding author: Yanling Health Center, Danyang, Jiangsu 212300, China. E-mail: yongxing_h338@126.com
There were 68 cases in this group, 49 males (72.1\%), 19 females (28.2\%), and the ratio is $2: 6: 1$; the age of onset is between 1.8-26 months, 8.5 month at average, $\leq 4$ months: 2 cases (5.9\% ), 4-10 months: 53 cases (77.9\% ), $\geq 10$ months: 13 cases $(19.2 \%)$; the time is $0.5-76 \mathrm{~h}$ from onset to treatment, $30 \mathrm{~h}$ at average, $\leq 24 \mathrm{~h}$ : 11 cases (16.2\%), 24-48 h: 47 cases $(69.1 \%), \geq 48$ h: 10 cases $(14.7 \%)$; 12 patients live in the city (17.6\%), and 56 patients live in the rural areas (82.4\%); first diagnosis as acute intussusception in 49 cases $(72.1 \%)$, first diagnosis as enteritis or bacillary dysentery in 16 cases (23.5\%), 3 cases of unknown diagnosis (4.4\%).

\subsection{Clinical manifestations}

Clinical manifestations were abdominal pain (paroxysmal crying) in 51 cases (75.0\%), vomiting in 59 cases (86.8\%), currant jelly blood stool (fecal occult blood positive) in 57 cases (83.8\%), pyocyte in stool convention in 48 cases (70.6\%), abdominal mass in 46 cases (67.6\%), and the symptoms included three or more of the above five terms in 55 cases $(80.9 \%)$.

\subsection{Verification methods}

All the 68 cases were accurately diagnosed by air enema reduction. Air enema was the preferred method for diagnosis and therapy of pediatric acute intussusception. The patients should lie on his/her back, and be inserted dou- 
ble lumen balloon catheter into the anus. The depth was about $5.0 \mathrm{~cm}$, and the air bags should be injected into air about $15-20 \mathrm{~mL}$. Under the condition of X-ray, the patient should first undergo diagnostic air enema under low pressure $(60 \mathrm{mmHg})$. If there was a "cup mouth-like" shadow in the head of the air column, it could be diagnosed as intussusception.

\subsection{Treatment methods}

All the patients in 68 cases underwent air enema reduction, and after the failure, turned to surgical reduction. In 15 cases, the patients underwent bowel resection due to intestinal necrosis $(9.6 \%)$.

\section{Results}

After all the patients in 68 cases were confirmed by diagnostic air enema as intussusception, the air bags were pressed gradually to 70-100 mmHg. The "cup mouth-like" shadow retreated to the right lower abdomen and disappeared in 35 cases, a great deal of gas entered into the small intestine, and the reduction was successful ( the success rate was 51.5\%); in 33 cases, the reductions were not successful after 2-3 times of air enema, so they turned to surgical reduction, and got successful ( the success rate is $48.5 \%)$; in 31 cases, the surgical reduction was successful without the intestinal necrosis, accounted for $45.6 \%$; bowel resection and Period I intestinal anastomosis in 2 cases, accounted for $2.9 \%$; in 3 cases, the incisions were infected after the surgical operation (4.4\%), and in 1 case, the incision split (1.5\%). No death occurred.

\section{Discussion}

\subsection{Pathogeny}

Acute intussusception refers to a kind of intestinal obstruction in which a part of the intestines and the corresponding mesentery are plugged into adjacent enteric cavity. It was the most common pediatric disease in childhood, and divided into primary and secondary intussusception. All the cases were primary intussusception in the hospital. The causes of acute intussusception were not clear yet, but related to virus infection, the dietary changes and intestinal spasm, genetic factors and somatostatin, gastrin, the anatomy with imperfect fixation of ileocecum, and the large extent of the ileocecum activities. Through 33 operation cases, the author predicted that the causes of pediatric acute intussusception might be still relative to the larger diameter of the ileocaecal valve and the poor reflux function of ileocaecal valve [2].

\subsection{Clinical manifestations}

Intussusception often occurs in children within 2 years old, especially between 4-10 months. In 68 cases, 53 children with 4-10 months accounted for $77.9 \%$. The clinical manifestations of intussusception included five symptoms, namely abdominal pain (paroxysmal crying), vomiting, currant jelly blood stool, stool pyocyte and abdomen mass. The clinical manifestations included three or more than three symptoms in 55 cases (80.9\%), and it was not so difficult to diagnose, so 49 cases were diagnosed as intussusception at first diagnosis. But still 16 cases were diagnosed as enteritis or bacillary dysentery, 3 cases of unknown diagnosis, accounted for $27.9 \%$. It indicated that some clinicians have not got sufficient knowledge about pediatric acute intussusception. The researcher believed that pediatric intussusception mostly belonged to entire intestinal obstruction. Before onset, the patients usually got diarrhea. If the patients cry severely (or dysphoria) or vomit after several days' diarrhea, and then the diarrhea stops, while several hours later, they had blood stool and pyocyte stool, namely severe crying (or irritability) diarrhea stops, blood stool and pyocyte stool, people should be highly alerted to these symptoms, and take timely air enema to make accurate diagnosis $[3,4]$.

\subsection{Air enema}

Air enema was accurate in diagnosis of intussusception with high reduction rate. In this group, the reduction rate was $51.5 \%$, but under the reported $90 \%$. The reasons might be that the patients' parents, clinicians have not got enough knowledge about the intussusception, which lead to the delay of treatment, with low rate of air enema reduction.

The experience of air enema: (1) Before the enema, the patients should be antispasmodic and sedative, the stomach and intestine were decompressed, the stomach was emptied, to prevent vomiting and sucking mistakenly during the enema; establish venous channels, correct dehydration and shock actively. (2) There were more sticky stools in some patients' colons, and sometimes they could block the balloon catheter. It was difficult to inject air by the enema machine, so used the air bags. It was easier to press the air bags to inject the air, but slow, and should pay closely attention to the extension of the colon to avoid the intestinal split due to the high pressure [5]. (3) During the process of enema, sometimes the air in the intestinal cavity would exhaust from the urinal pipe. Under X-ray, there was a V-shaped transparent zone around the urinal pipe. In this case, the pressure cannot be maintained in the intestinal cavity, and it was not easy to make reduction successfully. If the patients' parents can use their right hands to press the urinal pipe from the hip tissue to prevent the air exhausting around the urinal pipe, and ensure the pressure in the intestinal cavity, it was helpful for successful reduction. (4) The pressure of enema should not exceed 100 $\mathrm{mmHg}$ according to the textbook in order to prevent the colon splitting, and reduce the medical disputes. (5) X-ray observation of reduction: the shadow disappeared, and the air in the colon mostly entered into the small intestines to make them inflating quickly, and the reduction was successful. If the air in the colon entered into the small intestines and the bubbles were in rosary shapes, the enema 
was not successful, and the injection was still needed. In 1 case, when taking the enema, the air was in rosary shape, and the doctors thought the reduction was successful and stopped taking the enema. But the patients' symptoms did not disappear. They took the enema again, and it was diagnosed as the intussusception, which was the result of the first unsuccessful enema reduction. (6) The observation of successful reduction: The patients were quiet, and in short time, defecating yellow or green stool can further confirm the successful reduction, so no need to inject charcoal from the tube. (7) When the pressure reached $100 \mathrm{mmHg}$ for 2-3 times, the head no longer moved or exhibited "spring sign", the enema should be stopped, and prepared to take operation. The patients with the symptoms of blood stool in more than $48 \mathrm{~h}$, severe shock and abdominal distension and poor general conditions, should undergo lowpressure enema through remedial infusion and improving general conditions, and take timely operation after accurate diagnosis.

\subsection{Surgery}

The success rate of surgical reduction is also high. 33 cases in the hospital took successful surgical reduction, accounted for $48.5 \%$. 2 cases took bowel resection due to intestinal necrosis, and Period I intestinal anastomosis, accounted for $2.9 \%$. No intestinal leakage or death.

The experience of surgery: (1) Before the surgery: actively rectify dehydration and shock; check liver and kidney functions, electrolytes, blood coagulation functions; blood preparation; make good preoperative preparation. (2) Choose ketamine as the anaesthetic, plus part infiltration anesthesia for abdominal incision (skin, muscularis, peritoneum), to reduce the dose of panplegia medicine, lower the risks of accidents, and promote the recovery from surgery. (3) Children's napes were thin, and the abdominal skin's elasticity was good. So the incision in skin should not be too long, about $5.0 \mathrm{~cm}, 8.0-10 \mathrm{~cm}$ if it was appropriate, to expose the surgery, including the incision of the intestines. (4) The difficulties of surgical reduction were in the splenic flexure and ileocaecal valve. The surgical incision was not long, and in the right abdomen. The splenic flexure was far away from the incision, in acute angle. The angle would be much smaller by conventional extrusion method, so it was difficult for the head to pass. If pushed the head by using the index and middle fingers to the direction of left upper napes, making the splenic flexure into the shape of obtuse angle, the head would easily pass the splenic flexure when squeezing. It was the most difficult when the head passed the ileocaecal valve. If the assistant gently straightened the ileum terminal when the surgeon extended the ileocaecal valve from the neck of the intussusception by using the finger, the surgeon then squeezed from the other end of the head and caecum, the success rate of reduction can be enhanced significantly, which can reduce the intestinal excisions. Sometimes there would be lacerations on the colon ascendens serosa, just remedy them. In this way, it avoided intestinal excisions in 3 cases, and in 1 case, only the end of the intestines was excised, reserving the ileocaecal valve. The author believed that in the surgery, reset the intussusception as far as possible, take intestinal excision only when the intestinal necrosis was discovered, and avoild the right-half colon excision before the reduction [6]. (5) When the patients had already underwent intestinal decompression and intestinal excision, relaxation suture should be used to prevent the incisions infectious and splitting; no relaxation suture in pure reduction, the napes can be wrapped up by using the bandage, and usually there were no incision infectious or split.

\subsection{Preventions}

In 68 case, 56 patients live in rural areas (82.4\%), which is significantly more than those in cities-12 cases (17.6\%); the number of the cases with stool pyocyte (48 cases, $70.6 \%$ ) is higher than that of non-stool pyocute (20 cases, $29.4 \%$ ). It indicated that the life environment and hygiene conditions are relatively worse, and the intestines are sensitive to infection, which are the important causes of the high incidence of pediatric acute intussusception in rural areas. Therefore improving the life environment and hygiene conditions, enhancing the life quality can reduce the incidence of pediatric intussusception to a certain degree.

\section{References}

1. She Y, Tong E. Pediatric Surgery. The Third Edition. Beijing: People's Medical Publishing House. 1997;4.

2. Wang H, Li Z, Wu Z, etc.. Pediatric Acute Intussusception with Adenovirus Infection. Chinese Journal of Pediatric Surgery. 1988;9(3):156-159.

3. Li Z, Wang H, Ji Shi. Editor-in-Chief. Practical Pediatric Surgery. Beijing: People's Medical Publishing House. 2001:738-739.

4. Li X, Zhang J. Plasma somatostatin levels in intussusception. Chinese Journal of Pediatric Surgery. 2000;12(1):40-43.

5. Jin X, Chen W, Lei P, etc. Measure and observe pediatric intussusception serum gastrin. Chinese Journal of Pediatric Surgery. 1990;11:327.

6. Zhang J. Childrens Intussusception-spasm Theory. Journal of Clinical Pediatric Surgery. 2002;14(5):289-291. 